\title{
Cutaneous vasculitis as the sole manifestation of disseminated gonococcal infection: case report
}

\author{
M Mastrolonardo, F Loconsole, A Conte, F Rantuccio
}

The Department of Dermatology, University of Bari, Bari, Italy M Mastrolonardo

F Loconsole A Conte

F Rantuccio

Address for correspondence: Pr. Francesco Rantuccio viale Ennio 15 - 70124 Bari, Italy.

Accepted for publication 4 November 1993
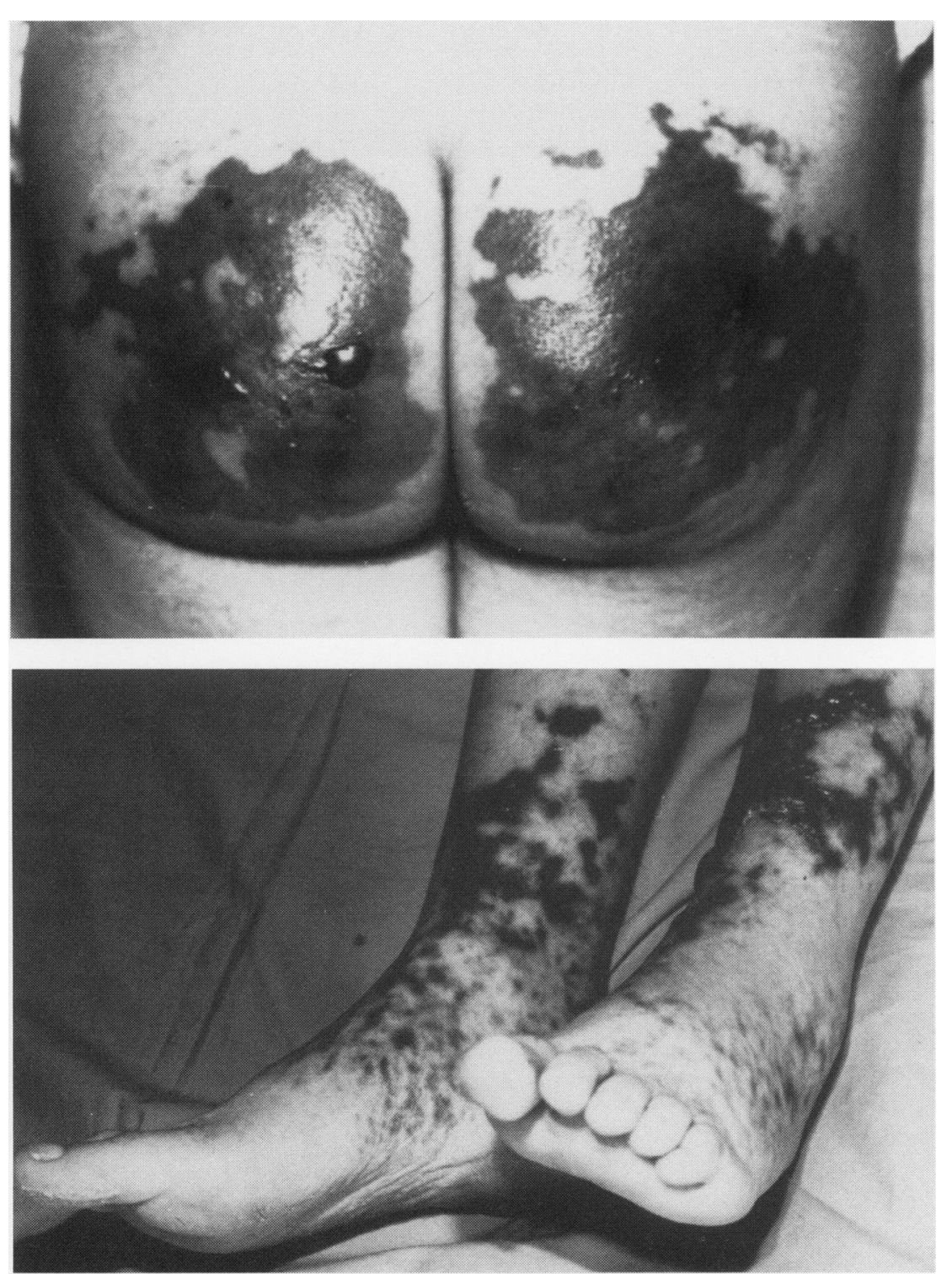

Figure Extensive, vesicobullous, haemorrhagic, and necrotic lesions located on the buttock (above) and lower limbs (below).

\begin{abstract}
One of the possible systemic complications of gonorrhoea is disseminated gonococcal infection (DGI), which is usually characterised by both skin and joint lesions. While joint involvement ranges from tenosynovitis to suppurative arthritis, cutaneous involvement features varied non-specific patterns often clinically and histologically consistent with vasculitis. We report a case of DGI in which an extensive, vesicobullous, haemorrhagic, and necrotic cutaneous vasculitis was the sole manifestation of the disease.
\end{abstract}

(Genitourin Med 1994;70:130-131)

\section{Case report}

A woman aged 24 years attended the Department of Dermatology and Sexually? Transmitted Diseases of the University of Bari with a one week history of rapidly enlarging, almost asymptomatic, vesicobul- $\widehat{\varnothing}$ lous, haemorrhagic, and necrotic lesions on the buttocks and lower limbs (fig). Her history was non-contributory with the exception? of three consecutive miscarriages during the $\vec{\omega}$ last four years. The eruption had not beeno heralded or accompanied by any constitu-? tional upset. Symptoms and signs of genitalo tract or joint involvement had been absent as? well. Finally, no local or general therapeutic $\vec{\omega}$ attempt had been made before the patient'so attendance at our clinic.

On physical examination, the patient was in otherwise good health. A full urine and blood chemistry panel failed to reveal any abnormality other than moderate hypocomplementaemia and mildly increased ESR, alpha $_{2}$ - and gammaglobulins, and circulating immune-complexes. A biopsy specimen was also taken from the border of one lesion and the results of light microscopy and $\operatorname{direct}_{\mathscr{L}}^{\mathbb{D}}$ immunofluorescence examinations confirmed $\overrightarrow{\overline{\hat{O}}}$ the clinical diagnosis of vasculitis.

A variety of screening tests were then performed in order to provide clues as to the? nature of the cause of vascular lesions. As is? our rule, the survey included detection of genital and non-genital foci. While examination of Gram stained smears from urethra, cervix, rectum, and throat gave negative results, cultures on Thayer-Martin medium? grew colonies of $N$ gonorrhoeae from the cervix. In contrast, all other investigations, including parallel tests for other sexuallyo transmitted diseases, human immunodefi- $N$ ciency virus (HIV) antibody titres determination, complete cardiological, rheumatological, otorhinolaryngological, odontological, ando neurological surveys, failed to reveal any con-क current abnormality.

Furthermore, a supplementary focused ${ }^{\circ}$ history was taken and revealed that the patient had had a stable heterosexual rela- $\frac{}{\Phi}$ tionship for the last 5 years and that she had음 never suffered from sexually transmitted diseases. She persuaded her 28-year-oldo husband to undergo STD investigations. He was asymptomatic and denied having hado other sexual partners. Nevertheless, specimens were taken from his urethra, rectum, and throat. Both Gram stained smears and cultures detected $N$ gonorrhoeae in the urethra. Finally, before starting penicillin therapy, to which the isolates from both 
patients showed highly susceptible, repeated aerobic and anaerobic cultures from blood and skin lesions were performed. Although results were constantly negative, the diagnosis of disseminated gonococcal infection was made. The dermatosis improved dramatically under 1 week of penicillin $G$ treatment.

\section{Discussion}

Sequelae of gonorrhoea include disseminated gonococcal infection (DGI). This peculiar pathological entity usually presents with two different clinical pictures which have been thought to be the expression of a pathological continuum where a "bacteriaemic" stage and a "joint sepsis" stage follow each other. ${ }^{1}$ The first is characterised by polyarthralgia, tenosynovitis and dermatitis; the second consists of purulent arthritis, more frequently without dermatitis..$^{2-4}$ As regards cutaneous involvement, diverse non-specific patterns often clinically and histologically consisting with vasculitis have been described. ${ }^{5-7}$

One of the main obstacles to diagnosis of DGI is the difficulty in obtaining microbiological confirmation. Skin, blood and synovial fluid cultures are often negative. ${ }^{238}$ However, evidence of a possible role played via immunological mechanisms by nonviable bacterial components have been reported..$^{59-12}$

According with the criteria established by Masi and Eisenstein in $1981,{ }^{13}$ we believe that the case here presented belongs to the broader category of "documented" DGI in which cutaneous involvement parallels the proof of gonococcal infection at a primary focus. In our opinion our case seems worth presenting as only a few cases have been previously reported in which dermatitis was the sole manifestation of the disease. ${ }^{3713}$ In the great majority of cases skin lesions, when present, coexist with evidence of joint involvement and/or other manifestations of gonococcal sepsis.
Finally, on the basis of our experience with vasculitis, ${ }^{14}$ we would now incidentally underline that the case described above is the only one in which to our knowledge gonococcal infection led to DGI. This finding obviously contrasts with those provided by other authors ${ }^{17}$ who have reported disseminated gonococcal infection to develop in 0.4 to $3 \%$ of patients with mucosal infection. In our opinion, this circumstance may be at least partially due to the high proportion of patients with asymptomatic primary infections $^{2}$ who therefore do not present for treatment at STD departments.

1 Keiser H, Ruben Fl, Wolinsky E, Kushner I. Clinica forms of gonococcal arthritis. $N$ Engl $\mathcal{f}$ Med 1968 ; 279:234-40.

2 Holmes KK, Counts GW, Beaty HN. Disseminated gonococcal infection. Ann Intern Med 1971;74:979-93.

3 O'Brien JP, Goldenberg DL, Rice PA. Disseminate gonococcal infection: a prospective analysis of 49 ponients and a revie patients and a review of pathophysiology and immu

4 Moyle G, Barton SE, Midgley J, Rowe IF, Keat AC L in a man with HIV infection. Genitourin Med 1990;66:91-2.

5 Shapiro L, Teisch JA, Brownstein MH. Dermatohistopathology of chronic gonococcal sepsis. Arch Dermatol 1973;107:403-6.

6 Eisenstein BI, Masi AT. Disseminated gonococcal infection (DGI) and gonococcal arthritis (GCA): I. bacteriology, epidemiology, host factors, pathogen factors, and pathology. Sem Arthritis Rheum 1981;10:155-72.

7 Barr J, Danielsson D. Septic gonococcal dermatitis. BMF 1971;1:482-5.

8 Al-Suileman SA, Grimes EM, Jonas HS. Disseminated gonococcal infections. Obstet Gynecol 1983;61:48-51.

9 Danielsson D, Michaelsson G. The gonococcal dermatitis syndrome. Acta Derm Venereol (Stockh) 1966;46: 257-61.

10 Kahn G, Danielsson D. Septic gonococcal dermatitis. Arch Dermatol 1969;99:421-5.

11 Walker LC, Ahlin TD, Tung KSK, Ahlin T, Williams R Circulating immune complexes in disseminated gonococcal infection. Ann Intern Med 1978;89:28-33.

12 Bayer AS. Gonococcal arthritis syndromes. An update on diagnosis and management. Postgrad Med 1980;67: 200-8.

13 Masi AT, Eisenstein BI. Disseminated gonococcal infection (DGI) and gonococcal arthritis (GCA): II. Clinical manifestations, diagnosis, complications, treatment, and prevention. Sem Arthritis Rheum 1981;10:173-97.

14 Mastrolonardo M, Loconsole F, Conte A, Rantuccio F. Le vasculiti ad espressione cutanea: osservazioni su 1167 casi osservati in un ventennio. G Ital Dermatol Venereol 1993;128:37-44. 